\title{
Inhibition of Bone Matrix Formation, Mineralization, and Resorption in Thyroparathyroidectomized Rats
}

\author{
J. Wergedal, M. Stauffer, D. Baylink, and C. Rich \\ From the University of Washington School of Medicine, Seattle, Washington \\ 98195, and the Veterans Administration Hospital, Seattle, Washington 98108
}

A в S T R A C T In previous work we found that vitamin D-deficient and also calcium-deficient rats developed hypocalcemia and an impairment of bone formation and mineralization. The present study of thyroparathyroidectomized (TPTX) rats was undertaken to determine the effect of hypocalcemia without secondary hyperparathyroidism. TPTX rats fed a normal diet developed hypocalcemia and hyperphosphatemia in association with impairment of osteoblastic bone matrix formation and of mineralization of newly formed matrix. The serum calcium $\times$ phosphorus product was not decreased. The decreased formation was largely due to a reduction in matrix apposition indicating decreased synthetic activity of individual osteoblasts. In contrast to the above results, when TPTX rats were fed a high-calcium diet to prevent hypocalcemia, no impairment of either formation or mineralization was found. From the results of these two experiments, it is reasonably certain that hypocalcemia was responsible for the inhibition of formation and mineralization. Moreover, based on the magnitude of the changes in serum calcium and bone parameters in TPTX rats, hypocalcemia could have accounted for the inhibition of formation and mineralization in calcium-deficient as well as vitamin D-deficient rats.

In TPTX rats the mineralization defect was manifested by decreases in both the rate of osteoid maturation (indicating a delayed onset of mineralization) and the rate of mineralization. A strong correlation $(r=$ $0.95, P<0.001$ ) was observed between these two rates suggesting a tight coupling of these two aspects of mineralization.

TPTX rats also had lower bone resorption rates and higher serum phosphorus levels than sham-operated ani-

A preliminary report of this work was published in abstract form in 1970 Clin. Res. 18: 143.

Dr. Baylink is recipient of Research Career Development Award DE 19108.

Received for publication 30 August 1972 and in revised form 4 January 1973. mals when the normal calcium diet was fed but not when the high-calcium diet was fed. Thus the inhibition of bone resorption in TPTX rats was at least partially prevented by correction of hyperphosphatemia. This is consistent with previous work showing an inverse relationship between serum phosphorus and bone resorption. Accordingly, the depression of bone resorption in TPTX rats was probably due to hyperphosphatemia as well as to hypoparathyroidism.

\section{INTRODUCTION}

In a previous study we found that vitamin $\mathrm{D}$ deficiency resulted in an inhibition of osteoblastic bone matrix formation and an impairment of matrix mineralization (1). The factor (s) responsible for these changes could not be established because in addition to having vitamin D deficiency, the animals were hypocalcemic and thus presumably had secondary hyperparathyroidism.

The possibility that hypocalcemia was responsible for the inhibition of formation and mineralization was supported by the finding of strong correlations between the rates of formation and mineralization and serum calcium but not phosphorus (1), and also by the results of a subsequent study on calcium-deficient animals. Rats fed a diet deficient in calcium but adequate in vitamin D developed hypocalcemia in association with an inhibition of formation and mineralization of similar magnitude to that observed in vitamin D-deficient animals (2). These results suggested that hypocalcemia, or hypocalcemia and secondary hyperparathyroidism, were responsible for the inhibition of formation and mineralization.

If hypocalcemia were the cause of these changes, one would expect to find similar changes in hypocalcemic, thyroparathyroidectomized (TPTX) ${ }^{1}$ rats. Accordingly,

\footnotetext{
${ }^{1}$ Abbreviations used in this paper: $\mathrm{PTH}$, parathyroid hormone; TPTX, thyroparathyroidectomized.
} 
the present study was undertaken to quantitate the effects of hypocalcemia without secondary hyperparathyroidism on matrix formation and mineralization. In addition, an attempt was made to assess the cause of the inhibition of osteoclastic bone resorption seen in TPTX rats.

\section{METHODS}

\section{Protocol}

Male weanling (22-day old) Sprague-Dawley rats were randomly divided into two groups, both fed a semisynthetic diet containing $0.6 \%$ calcium and $0.6 \%$ phosphorus (3). Prior to the experimental period, one group was thyroparathyroidectomized (TPTX) by blunt dissection and the other group was sham-operated. The TPTX rats were injected every other day with $4 \mu \mathrm{g} / 100 \mathrm{~g}$ body weight of L-thyroxin (Levoid; Nutritional Control Products, Hollywood, Fla.); and the sham-operated group was injected with an equal volume of diluent. 3 days after TPTX, blood samples were drawn from the tail fur serum calcium analysis. Only those TPTX rats with serum calcium values of less than 9.0 $\mathrm{mg} / 100 \mathrm{ml}$ were used for the study.

The rationale for using the protocol given below to make quantitative histological measurements of bone parameters has been described in detail elsewhere $(1,4,5)$. When 27 days of age both the sham-operated and TPTX rats were divided into two groups of nine rats each (i.e., basal and final groups). The two basal groups were sacrificed at the start of the experimental period, $2 \mathrm{~h}$ after i.p. injections of $20 \mathrm{mg}$ tetracycline per $\mathrm{kg}$ body weight. The final groups were injected with $10 \mathrm{mg}$ tetracycline per $\mathrm{kg}$ body weight i.p. daily for 7 days beginning at the time of sacrifice of the basal groups. All rats were sacrificed 10 days after starting the daily tetracycline injections and $2 \mathrm{~h}$ after a final injection of tetracycline, $20 \mathrm{mg} / \mathrm{kg}$ body weight i.p., to label the mineralizing front (1). Since a preliminary experiment indicated that food consumption was slightly decreased in TPTX rats, in this study the intact rats were pair fed with the TPTX rats to avoid effects of differences in food consumption on bone parameters.

A second experiment was done using a protocol very similar to that described above, the major difference being that both the sham-operated group and the TPTX final groups received a diet containing $1.2 \%$ calcium and $0.55 \%$ phosphorus throughout the experimental period. We previously demonstrated that this diet usually results in normal values of serum calcium and phosphorus in TPTX rats (5). In this second experiment, the rats were 28 days old when tetracycline labeling was started and there were 10 rats each in the basal and final control groups and 15 rats each in the basal and final TPTX groups.

In a separate experiment the diurnal variation in serum calcium and phosphorus was determined in 28-day old TPTX rats fed a diet containing $1.2 \%$ calcium and $0.55 \%$ phosphorus. Serum measurements were made starting three days after the animals were given a $1.2 \%$ calcium and $0.55 \%$ phosphorus diet.

\section{Serum chemistries}

Blood was obtained by cardiac puncture at sacrifice or from tail vein when a series of determinations was made on the same animals. Total serum calcium and phosphorus determinations were made as previously described (1), and ionized calcium was measured on freshly obtained serum by means of an Orion Model 98-20 flow through electrode system (Orion Research, Inc., Cambridge, Mass.) (6).

\section{Bone parameters}

At sacrifice, the tibias were removed for analysis. Specific sampling sites, section preparation, methods of analysis and calculations have been treated in detail elsewhere $(1,4,5)$. Provided below are definitions of the calculated bone parameters, each of which is a measure of a discrete process.

Periosteal matrix formation rate $\left(\mathrm{mm}^{3} /\right.$ day). This includes all periosteal matrix, mineralized matrix as well as osteoid, deposited during the experimental period. It is identical to the bone formation rate, except when osteoid maturation is impaired, in which case it exceeds the bone formation rate.

Periosteal matrix apposition rate $(\mu m / d a y)$. This is the width of new periosteal matrix added per day and includes both osteoid and mineralized matrix.

Periosteal osteoid maturation rate $(\% / h)$. This is a measure of the onset of mineralization and is calculated by considering that osteoid is $0 \%$ mature when deposited and $100 \%$ mature when mineralization is initiated. The term "maturation" is used because we have demonstrated that chemical changes occur in osteoid prior to the onset of mineralization (7). Osteoid maturation rate is probably a better measurement of the onset of mineralization than is osteoid width since an increase in osteoid width may be related to either an increase in matrix apposition or a delay in the onset of mineralization, whereas the osteoid maturation rate is independent of apposition (1).

Periosteal initial mineralization rate (\% of maximum/h). This is a measure of the rate at which mineral concentration increases from 0 to $20 \%$ of maximum mineral concentration. This rate is expressed as percent of maximum mineral concentration in mature bone but does not apply to mineral deposition between 21 to $100 \%$ of maximum mineral concentration. This method, which is based on the distance that tetracycline diffuses into low mineral content bone, gives results similar to those obtained from measurements of the actual rates of calcium and phosphorus deposition made by means of electron microprobe (8).

Endosteal bone resorption rate $\left(\mathrm{mm}^{3} /\right.$ day $)$. Since resorption increases, whereas formation decreases medullary area, the resorption rate is equal to the mean daily change in medullary area plus the endosteal bone formation rate $(1,9)$. In our sampling site in the tibial diaphysis, the endosteal resorption rate represents essentially the total resorption rate because no resorption occurs at the periosteum, and that occurring at vascular canals is only $10 \%$ of the total and was ignored in this study (9).

Linear rate of endosteal bone resorption $(\mu m / d a y)$. This is the mean width of endosteal bone resorbed per day and is calculated by dividing the amount of bone resorbed by the length of the endosteal surface involved in resorption. It is analogous to the periosteal matrix apposition rate.

\section{RESULTS}

TPTX rats fed our control diet containing $0.6 \%$ calcium and $0.6 \%$ phosphorus developed hypocalcemia and hyperphosphatemia (Table I). Serum calcium in the basal and final TPTX groups were similar suggesting that the degree of hypocalcemia was sustained throughout the 10day experimental period (Table I). In these TPTX rats as compared with sham-operated, intact control rats, 
TABLE I

Serum and Bone Parameters in Intact* and TPTX Rats Fed a $0.6 \%$ Calcium and a $0.6 \%$ Phosphorus Diet

\begin{tabular}{lcc}
\hline & Intact & TPTX \\
\hline Body weight, $g$ & $92.0 \pm 3.0 \ddagger$ & $92.0 \pm 10.0$ \\
Serum calcium, $m g / 100 m l$ (basal & & \\
$\quad$ group) 8 & $9.7 \pm 0.2$ & $6.6 \pm 1.0 \|$ \\
Serum calcium, $m g / 100 m l$ & $10.4 \pm 0.4$ & $7.2 \pm 1.0 \|$ \\
Serum phosphorus, $m g / 100 m l$ & $10.0 \pm 0.7$ & $15.9 \pm 2.9 \|$ \\
Serum calcium $\times$ phosphorus & & \\
$\quad(m g / 100 m l)$ & $104.0 \pm 5.0$ & $113.0 \pm 15.0$ \\
Periosteal osteoid width, $\mu m$ & $6.1 \pm 0.7$ & $8.3 \pm 1.59$ \\
Periosteal mineral front width, $\mu m$ & $5.6 \pm 0.8$ & $7.9 \pm 1.1 \|$ \\
Total area, $m m^{2}$ & $2.70 \pm 0.12$ & $2.56 \pm 0.12 * *$ \\
Medullary area, $m m^{2}$ & $1.05 \pm 0.07$ & $0.87 \pm 0.06 \|$ \\
Periosteal surface, $m m$ & $5.96 \pm 0.17$ & $5.85 \pm 0.17$ \\
Endosteal forming surface, $m m$ & $2.62 \pm 0.41$ & $2.39 \pm 0.43$ \\
Endosteal resorbing surface, $m m$ & $1.57 \pm 0.48$ & $1.78 \pm 0.12$ \\
Endosteal resorbing surface, $\%$ & $37.2 \pm 9.8$ & $43.1 \pm 4.9$ \\
& & \\
\hline
\end{tabular}

* Sham-operated.

$\ddagger$ Mean \pm SD.

$\S$ All values are for final group with this exception.

$\| P<0.001$.

If $P<0.005$.

** $P<0.05$.

osteoblastic matrix formation, the mineralization of newly formed matrix, and the osteoclastic bone resorption rate were all depressed (Table II). Because of the mineralization defect, bone formation was depressed more than matrix formation.

The matrix formation rate is a function of two components, the forming surface and the matrix apposition rate; and the inhibition of matrix formation in the TPTX rats was almost exclusively due to a decreased matrix apposition rate indicating decreased synthetic activity of individual osteoblasts (Table II).

In the TPTX rats there was a delayed onset of mineralization, as indicated by the impairment of osteoid maturation, and a decreased mineralization rate (Table II). These changes are independent of matrix apposition and are typical of those seen in osteomalacia (1). Thus, despite adequate intake of vitamin $\mathrm{D}$, calcium, and phos- phorus, these TPTX rats developed a mineralization defect.

Of particular interest was the finding of a strong correlation between the rates of osteoid maturation and mineralization when the data from the sham-operated control rats and the TPTX rats were pooled (Fig. 1). Although the slopes for the sham-operated and TPTX groups may differ, it is clear that a relationship exists over a wide range of rates. This relationship cannot be explained by the manner in which these rates were determined since they represent independent measurements. These data suggest that the onset of mineralization and the rate of mineralization are tightly coupled, a conclusion consistent with previous observations (1-5).

In TPTX rats as compared with intact rats, there was a $70 \%$ decrease in bone resorption (Table II). Bone resorption is a function of two components, the resorbing surface and the linear rate of bone resorption. In the TPTX rats the decrease in resorption was largely due to a decrease in the linear rate of bone resorption.

When TPTX rats were fed a $1.2 \%$ calcium and a $0.55 \%$ phosphorus diet, rather than the $0.6 \%$ calcium and $0.6 \%$ phosphorus diet, only slight changes in serum calcium and phosphorus in bone were found (Tables III and IV). In these TPTX rats as compared with shamoperated control rats fed the same diet, serum calcium was decreased only about $10 \%$ and serum phosphorus was essentially unchanged (Table III).

In sharp contrast to the first experiment, in these TPTX rats neither matrix formation nor mineralization were decreased (Table IV). In addition, whereas in the first experiment bone resorption was markedly depressed in the TPTX rats, in this experiment bone resorption was similar in TPTX and sham-operated control rats fed the same diet (Table IV). These two experiments show that the inhibition of matrix formation and mineralization and bone resorption seen in hypocalcemic hyperphosphatemic TPTX rats can be largely corrected

TABLE II

Bone Matrix Formation and Mineralization and Bone Resorption in Intact* and TPTX Rats Fed a $0.6 \%$ Calcium and a $0.6 \%$ Phosphorus Diet

\begin{tabular}{|c|c|c|c|c|}
\hline & Intact & TPTX & $\begin{array}{c}\% \\
\text { Change }\end{array}$ & $P$ \\
\hline Periosteal matrix formation rate, $\mathrm{mm}^{3} / d a y$ & $0.055 \pm 0.007 \ddagger$ & $0.045 \pm 0.009$ & -18 & $<0.05$ \\
\hline Periosteal matrix apposition rate, $\mu m / d a y$ & $9.7 \pm 1.3$ & $8.1 \pm 1.5$ & -16 & $<0.05$ \\
\hline Periosteal osteoid maturation rate, $\% / h$ & $5.8 \pm 0.8$ & $3.6 \pm 0.7$ & -38 & $<0.001$ \\
\hline Periosteal initial mineralization rate, $\%$ of maximum $/ h$ & $1.4 \pm 0.1$ & $0.7 \pm 0.1$ & -50 & $<0.001$ \\
\hline Endosteal bone resorption rate, $m m^{3} / d a y$ & $0.020 \pm 0.008$ & $0.006 \pm 0.006$ & -70 & $<0.001$ \\
\hline Linear bone resorption rate, $\mu m / d a y$ & $10.4 \pm 4.9$ & $2.5 \pm 2.5$ & -76 & $<0.001$ \\
\hline
\end{tabular}

* Sham-operated.

$\ddagger$ Mean \pm SD. 
by feeding TPTX rats a diet which results in near normal levels of serum calcium and phosphorus.

\section{DISCUSSION}

The present study of TPTX rats is one of a series of investigations in which we have quantitated discrete bone processes associated with matrix formation, mineralization, and bone resorption. These studies have been aimed at identifying systemic factors that influence each of these bone processes under pathophysiologic conditions $(1,2,5)$.

The results of this study strongly implicate hypocalcemia as a factor that impairs matrix formation and mineralization. On a normal calcium diet, TPTX rats became hypocalcemic and rates of bone matrix formation, osteoid maturation and bone mineralization were inhibited (Table II). Evidence of decreased bone formation in both TPTX and parathyroidectomized animals and increased osteoid width in parathyroidectomized animals have been previously described (10-12). A1though an increase in osteoid width may be associated with an increase in bone matrix apposition $(1,13)$, in TPTX rats the wide osteoid was due to a delayed onset of mineralization, as indicated by a decreased rate of osteoid maturation. When the dietary calcium level was raised so that the TPTX rats were able to maintain near normal serum calcium levels, formation and mineralization were no longer impaired. Thus neither thyroparathyroidectomy per se, nor the absence of parathyroid hormone $(\mathrm{PTH})$ or calcitonin was responsible for the impaired matrix formation and mineralization. Impaired formation has also been found in the other conditions which resulted in hypocalcemia, i.e., calcitonin treat-

TABLE III

Serum and Bone Parameters in Intact* and TPTX Rats Fed a $1.2 \%$ Calcium and a $0.55 \%$ Phosphorus Diet

\begin{tabular}{lcc}
\hline & Intact & TPTX \\
\hline Body weight, $g$ & $112.0 \pm 3.0 \ddagger$ & $109.0 \pm 11.0$ \\
Serum ionized calcium, $\mathrm{mg} / 100 \mathrm{ml}$ & $4.7 \pm 0.2$ & $4.0 \pm 0.4 \S$ \\
Serum calcium, $\mathrm{mg} / 100 \mathrm{ml}$ & $10.5 \pm 0.4$ & $9.5 \pm 0.8 \|$ \\
Serum phosphorus, $\mathrm{mg} / 100 \mathrm{ml}$ & $13.3 \pm 1.2$ & $13.5 \pm 1.4$ \\
Serum calcium $\times$ phosphorus & & \\
$\quad(m g / 100 \mathrm{ml})^{2}$ & $140.0 \pm 13.0$ & $128.0 \pm 17.0$ \\
Periosteal osteoid width, $\mu m$ & $10.6 \pm 1.0$ & $11.5 \pm 1.09$ \\
Periosteal mineral front width, $\mu m$ & $5.7 \pm 0.4$ & $6.8 \pm 0.9 \|$ \\
Total area, $m m^{2}$ & $2.92 \pm 0.16$ & $2.97 \pm 0.22$ \\
Medullary area, $m m^{2}$ & $0.97 \pm 0.09$ & $0.90 \pm 0.12$ \\
Periosteal surface, $m m$ & $6.23 \pm 0.18$ & $6.27 \pm 0.24$ \\
Endosteal forming surface, $m m$ & $2.29 \pm 0.30$ & $2.28 \pm 0.54$ \\
Endosteal resorbing surface, $m m$ & $1.48 \pm 0.30$ & $1.46 \pm 0.50$ \\
Endosteal resorbing surface, $\%$ & $39.3 \pm 7.0$ & $39.1 \pm 13.0$ \\
\hline
\end{tabular}

* Sham-operated.

$\ddagger$ Mean \pm SD

$\S P<0.001$.

$\| P<0.005$.

I $P<0.05$.

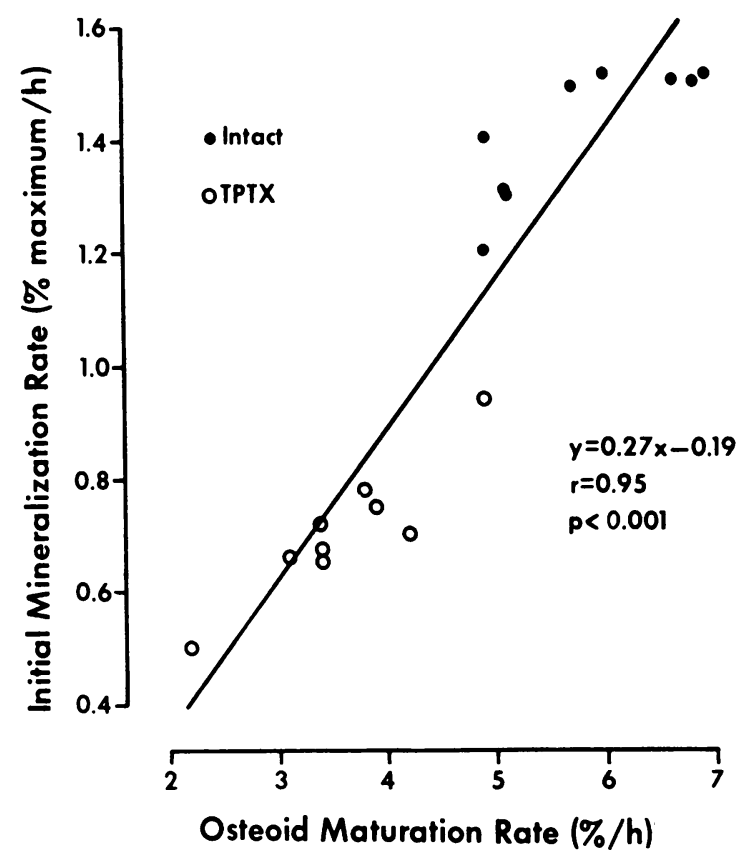

FIGURE 1 Relationship between the rates of osteoid maturation and mineralization in intact and TPTX rats together. Although the slopes for the intact and TPTX rats may differ, these data emphasize that a relationship exists between these two rates over a wide range of rates.

ment, vitamin $\mathrm{D}$ deficiency, and calcium deficiency $(1,2,9)$.

Hyperphosphatemia, though present in the TPTX animals, is an unlikely explanation for the depression of matrix formation and mineralization. For example, in hypocalcemic vitamine D-deficient rats both formation and mineralization were depressed even though serum phosphorus was normal (1). And, in vitro, reduced but

\section{TABLE IV}

Bone Matrix Formation and Mineralization and Bone Resorption in Intact ${ }^{*}$ and TPTX Rats Fed a $1.2 \%$ Calcium and $0.55 \%$ Phosphorus Diet

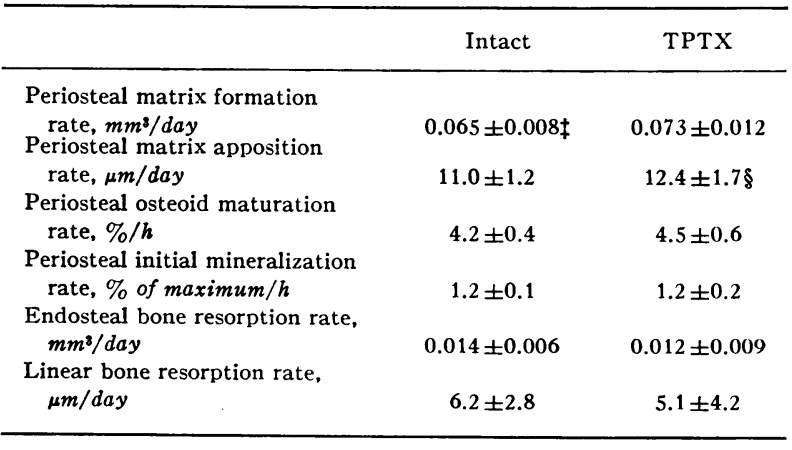

* Sham-operated.

$\ddagger$ Mean \pm SD.

$\& P<0.05$. 
TABLE V

Relationship between Serum Calcium and Phosphorus and

Bone Processes in TPTX, Calcium-deficient and Vitamin D-deficient Rats*

\begin{tabular}{|c|c|c|c|}
\hline & TPTX & $\begin{array}{l}\text { Calcium } \\
\text { deficient }\end{array}$ & $\begin{array}{c}\text { Vitamin D } \\
\text { deficient }\end{array}$ \\
\hline Serum calcium, $\% \Delta$ & $-32 \ddagger$ & -26 & -31 \\
\hline Serum phosphorus, $\% \Delta$ & +59 & +16 & +2 \\
\hline Periosteal matrix apposition, $\% \Delta$ & -16 & -37 & -25 \\
\hline Osteoid maturation rate, $\% \Delta$ & -38 & -55 & -50 \\
\hline Mineralization rate, $\% \Delta$ & -50 & -55 & -52 \\
\hline
\end{tabular}

* See references 1 and 2 for further data on vitamin D-deficient and calcium deficient animals, respectively.

$\ddagger \% \Delta$ from control group, each test group having its own control group; data used to calculate $\% \Delta$ were mean values for about a 10 day experimental period.

not elevated phosphorus in incubation medium inhibited radioproline incorporation into bone matrix (14). From these observations it is probable that hypocalcemia inhibits matrix formation, osteoid maturation, and mineral deposition.

The inhibition of the onset of mineralization in TPTX rats was associated with hypocalcemia but not a decreased serum calcium phosphorus product; this product was actually increased. The onset of mineralization appears to be dependent upon osteoid maturation. Accordingly, matrix changes in lipid concentration, acid phosphatase activity, and particularly proteinpolysaccharide concentration are coordinated both spatially and temporally with the onset of mineralization $(7,15,16)$. Because osteoid maturation implies that the onset of mineralization is at least partially under cellular control, it seems likely that hypocalcemia inhibits the onset of mineralization by adversely affecting cell-mediated processes.

The extent to which cellular activity is involved in calcium and phosphorus deposition once the process has been initiated is uncertain, though there is evidence of a functional membrane around bone, which is maintained by bone cells and which influences the ionic composition of bone water (17). In addition, the finding in animals treated with large amounts of fluoride, of a decreased mineralization rate despite normal levels of serum calcium and phosphorus, suggests that cellular activity may also be involved in this process (4). Thus, the inhibition of the mineralization rate by hypocalcemia may also be through an effect on cellular activity.

The possibility that hypocalcemia inhibits osteoid maturation and mineralization by a similar mechanism is consistent with the strong correlation between the rate of osteoid maturation and mineralization in the present study. Furthermore, the magnitude of the changes in these two rates was similar under a number of other experimental conditions, viz., fluoride treatment, vitamin D deficiency, calcium deficiency, and phosphorus deficiency $(1,2,4,5)$. Although the reason for this relationship is unknown, it is clear that these two rates are tightly coupled under a variety of experimental conditions.

It is generally held that the decrease in bone resorption in hypoparathyroidism is entirely due to PTH deficiency whereas evidence is accumulating to indicate that in TPTX rats hyperphosphatemia inhibits bone resorption. First, we previously found that in TPTX rats there is an inverse relationship between serum phosphorus and percent endosteal resorbing surface over serum phosphorus values ranging from high to low (5). Second, in the present study bone resorption was low in hyperphosphatemic but near normal in normophosphatemic TPTX rats (Tables II and IV). Third, in tissue culture increasing phosphorus concentration in the incubation medium decreases bone resorption and also decreases the effect of PTH to stimulate bone resorption (18). Thus, the decreased resorption in the TPTX rats was probably due to hyperphosphatemia as well as hypoparathyroidism. In view of this, it is doubtful that PTH determines $75 \%$ of bone turnover since this concept is based on the observation that turnover falls $75 \%$ after removal of the parathyroid glands (19).

Since the resorption rate was only slightly decreased in the normophosphatemic TPTX rats (Table IV), one might conclude that hyperphosphatemia, as opposed to hypoparathyroidism, was primarily responsible for the inhibition of bone resorption in the hyperphosphatemic TPTX rats (Table II). However, the control group for the experiment in Table IV was fed a high calcium diet which may have partially inhibited PTH secretion and thus PTH-mediated bone resorption. Accordingly, it is possible that the resorption rate would have been less in the normophosphatemic TPTX rats than in intact rats fed a normal calcium diet. In any case, it is evident that lowering the serum phosphorus was associated with an increase in bone resorption.

Although there is evidence that hypocalcemia per se stimulates bone resorption $(20,21)$, in this study hypocalcemia was associated with decreased, not increased resorption. Also in tissue culture studies, decreasing calcium concentration in the medium does not significantly enhance resorption (18).

Recent work suggests that the most potent vitamin D metabolite with respect to bone resorption is 1,25 -dihydroxycholecalciferol $\left(1,25-\mathrm{OH}_{2} \mathrm{D}_{3}\right)$ (22), and that the synthesis of this metabolite is PTH dependent (23). Accordingly, it is possible that some of the bone changes 
in TPTX rats results from an abnormality in vitamin D metabolism. If so, these changes were not evident in TPTX rats fed a diet which resulted in near normal levels of serum calcium and phosphorous (Tables III and IV).

A major reason for undertaking this study was to gain some insight as to the factors responsible for the inhibition of matrix formation and mineralization seen in vitamin D-deficient and also in calcium-deficient animals $(1,2)$. The relationships between serum and bone parameters in TPTX, vitamin D-deficient, and calcium-deficient rats are compared in Table $\mathrm{V}$. The changes in serum calcium and mineralization were similar in the three groups. However, on the basis of the degree of hypocalcemia, the inhibition of formation in the TPTX rats was less than expected. That these three experiments were done at different times and under conditions which were not identical may not be an adequate explanation for this discrepancy.

The degree of hyperphosphatemia was greatest in the TPTX rats, and this may have accounted for the lesser inhibition of bone formation (24). Alternately, the presence of secondary hyperparathyroidism as well as hypocalcemia may have been responsible for the relatively greater depression of matrix formation in the calciumdeficient and vitamin D-deficient rats (Table V). The validity of this possibility is uncertain at the present time, because under some conditions hyperparathyroidism results in decreased bone formation, whereas under other conditions it results in increased bone formation (13, 25-27).

This study emphasizes that changes in serum calcium and phosphorus have important effects on bone processes. It seems possible that the effects of the serum ion changes are mediated through changes in the ionic concentration of calcium and possibly phosphorus within bone cells. If so, the results of this study are consistent with the concept advanced by Rasmussen that intracellular calcium and phosphate ion concentration play an important role in bone cell metabolism (28). In this context, since a number of factors influence the concentration of calcium and phosphorus within cells, the similarities in Table $\mathrm{V}$ are more impressive than the differences.

\section{ACKNOWLEDGMENTS}

We are grateful to R. Haller for technical assistance, Mrs. K. Hashimoto for computer assistance, S. Baker for preparing the figures, and Mrs. S. Grimes for typing the manuscript.
This study was supported in part by National Institutes of Health Grants AM 09096 and HD-04872.

\section{REFERENCES}

1. Baylink, D., M. Stauffer, J. Wergedal, and C. Rich. 1970. Formation, mineralization, and resorption of bone in vitamin D-deficient rats. J. Clin. Invest. 49: 1122.

2. Stauffer, M., D. Baylink, J. Wergedal, and C. Rich. 1973. Decreased bone formation and mineralization, and enhanced resorption in calcium-deficient rats. $\mathrm{Am}$. J. Physiol. In press.

3. Wergedal, J. 1969. Enzymes of protein and phosphate catabolism in rat bone. I. Enzyme properties in normal rats. Calcif. Tissue Res. 3 : 55.

4. Baylink, D., J. Wergedal, M. Stauffer, and C. Rich. 1970. Effects of fluoride on bone formation, mineralization and resorption in the rat. In Fluoride in Medicine. T. L. Vischer, editor. Hans Huber, Bern, Switzerland. 37.

5. Baylink, D., J. Wergedal, and M. Stauffer. 1971. Formation, mineralization and resorption of bone in hypophosphatemic rats. J. Clin. Invest. 50: 2519.

6. Moore, E. W. 1970. Ionized calcium in normal serum ultrafiltrates, and whole blood determined by ion-exchange electrodes. J. Clin. Invest. 49: 318.

7. Baylink, D., J. Wergedal, and E. Thompson. 1972. Loss of proteinpolysaccharides at sites where bone mineralization is initiated. J. Histochem. Cytochem. 20: 279.

8. Baylink, D., and J. Wergedal. 1971. Effect of vitamin D-deficiency on the bone mineralization rate. Clin. Res. 19: 470 .

9. Baylink, D., E. Morey, and C. Rich. 1969. Effect of calcitonin on the rates of bone formation and resorption in the rat. Endocrinology. 84: 261.

10. Jowsey, J., R. E. Rowland, J. H. Marshall, and F. C. McLean. 1958. The effect of parathyroidectomy on haversian remodeling of bone. Endocrinology. 63: 903.

11. Burkhart, J. M., and J. Jowsey. 1966. Morphologic evidence of osteomalacia in the parathyroidectomized dog. Mayo Clin. Proc. 41: 663.

12. Kelly, P. J. 1971. Effects of thyroid and parathyroid deficiency on bone remodeling distal to a venous tourniquet. J. Anat. 110: 349.

13. Sherrard, D. J., D. J. Baylink, J. E. Wergedal, and N. A. Maloney. 1972. Increased bone formation in uremic patients. Clin. Res. 20: 193.

14. Raisz, L. G. 1970. The pharmacology of bone. Fed. Proc. 29: 1176 .

15. Baylink, D. J., and J. E. Wergedal. 1971. Effets du flour sur la mineralisation des os chez le rat. Med. Hyg. 29: 1554.

16. Wergedal, J. E., and D. J. Baylink. 1969. Distribution of acid and alkaline phosphatase activity in undemineralized sections of the rat tibial diaphysis. J. Histochem. Cytochem. $17: 799$.

17. Canas, F., A. R. Terepka, and W. F. Neuman. 1969. Potassium and milieu interieur of bone. Am. J. Physiol. 217: 117

18. Raisz, L. G., and I. Niemann. 1969. Effect of phosphate, calcium and magnesium on bone resorption and hormonal responses in tissue culture. Endocrinology. 85: 446.

19. Harris, William H., and Robert P. Heaney. 1969. Skeletal renewal and metabolic bone disease (concluded). $N$. Engl.J. Med. 280: 303. 
20. Rasmussen, H., C. Anast, and C. Arnaud. 1967. Thyrocalcitonin, EGTA, and urinary electrolyte excretion. J. Clin. Invest. 46: 746.

21. Waron, M., and C. Rich. 1969. Rate of recovery from acute hypocalcemia as a measure of calcium homeostatic efficiency in the dog. Endocrinology. 85: 1018.

22. Raisz, L. G., C. L. Trummel, M. F. Holick, and H. F. DeLuca. 1972. 1,25-dihydroxycholecalciferol: a potent stimulator of bone resorption in tissue culture. Science (Wash. D. C.). 175 : 768.

23. Garabedian, M., M. F. Holick, H. F. DeLuca, and I. T. Boyle. 1972. Control of 25-hydroxycholecalciferol metabolism by parathyroid glands. Proc. Natl. Acad. Sci.U.S. A. 69: 1673 .
24. Rasmussen, H., J. Feinblatt, N. Nagata, and M. Pechet. 1970. Effect of ions upon bone cell function. Fed. Proc. 29: 1190.

25. Flanagan, B., and G. Nichols, Jr. 1969. Bone matrix turnover and balance in vitro. I. The effects of parathyroid hormone and thyrocalcitonin. J. Clin. Invest. 48: 595.

26. Kalu, D. N., J. Pennock, F. H. Doyle, and G. V. Foster. 1970. Parathyroid hormone and experimental osteosclerosis. Lancet. $1: 1363$.

27. Sherrard, D., D. Baylink, and J. Wergedal. 1972. Bone disease in uremia. Transactions for the American Society of Artificial Internal Organs. 18: 412.

28. Rasmussen, H. 1971. Ionic and hormonal control of calcium homeostasis. Am. J. Med. 50: 567. 